\section{Supportive Care in Cancer Patients}

R ecent D evelopments

A ntibiotics and Chemotherapy, Vol. 50

M. Karthaus, A G anser

K arger, B asel, 2000. V III + 196 p., 9 Fig., 38 Tab., D M 189,-

ISB N 3-8055-6910-6

A uf fast 200 Seiten werden die häufigsten und für die Onkologie wesentlichen Begleit- und Präventivmaßnahmen zur $B$ ehandlung von Chemotherapie-induzierten unerwünschten Wirkungen unter Berücksichtigung von Ä tiologie, Pathophysiologie und $B$ eschwerdebild in $18 \mathrm{~K}$ apiteln differenziert dargestellt. D en Schwerpunkt bilden die antibakteriellen, antimikrobiellen und antimykotischen therapeutischen $\mathrm{Maß}$ nahmen bei Neutropenie und deren serologischer und molekularbiologischer Diagnostik unter Einschluß auch neuerer Entwicklungen der B ehandlung mit hämatopoetischen Wachstumsfaktoren. $D$ aneben werden die gängigen supportivmedizinischen $\mathrm{M}$ aßnahmen bei Mucositis, bei Venenkatheter-Komplikationen, bei zytostatikabedingten Paravasaten auch neuerer zytotoxischer Präparate, bei Emesis, Diarrhö und O bstipation abgehandelt. E in weiteres $K$ apitel ist der Pathogenese, Prävention und Therapie thromboembolischer Komplikationen bei Tumorerkrankungen gewidmet.

Den Autoren ist es gelungen, einen breiten A usschnitt der Supportivmedizin in Pathogenese, Diagnostik, Prävention und Therapie nach dem neuesten Stand verständlich darzustellen. D as B uch gibt einen U p-to-date-Ü berblick, nicht nur für O nkologen und $\mathrm{Hämatologen,} \mathrm{sondern} \mathrm{auch} \mathrm{für} \mathrm{alle} \mathrm{Betreuenden}$ von $\mathrm{K}$ rebspatienten.

R. $H$ artenstein, M ünchen

\section{Therapie gastrointestinaler Tumoren}

Prinzipien der $\mathrm{C}$ hirurgischen $\mathrm{K}$ linik und Poliklinik der Technischen U niversität M ünchen

J.D. Roder, H.J. Stein, U. Fink

Berlin, Springer, 2000, XV III +432 S., 127 z.T. farb. A bb., DM 129,-

ISB N 3-540-66189-1

M it dem anläßlich des 60. G eburtstags von Prof. D r.J.R . Siewert erschienenen Buch werden der aktuelle Standard der in D eutschland gültigen onkologischen Therapiekonzepte sowie Richtlinien in der Diagnostik gastrointestinaler Malignome dokumentiert. $D$ aneben werden auch die wissenschaftlichen Ergebnisse aus dem Patientengut der K linik dargestellt. Im Geleitwort von Prof. Dr. C. Herfarth wird die Bedeutung der interdisziplinären Z usammenarbeit der einzelnen Fachbereiche hervorgehoben, welche - wie im vorliegenden Buch dokumentiert - im K linikum rechts der Isar unter der Leitung von Prof. Dr. J.R. Siewert beispielhaft praktiziert wird.

Im ersten Teil des B uches werden die einzelnen diagnostischen und therapeutischen Verfahren/M ethoden allgemein besprochen, gefolgt vom zweiten speziellen Teil, welcher dem eigentlichen Schwerpunkt der Klinik, den gastrointestinalen Tumoren, gewidmet ist. $\mathrm{N}$ ach einer E inleitung über die B edeutung des exakten Stagings für individualisierte Therapiekonzepte folgt eine detaillierte $\mathrm{A}$ bhandlung aller diagnostischen Verfahren. $\mathrm{N}$ eben den konventionellen radiologischen Verfahren wie Röntgendiagnostik, CT, MRT und nuklearmedizinischen M ethoden (PET-Scan) wird auch ein kurzer A usblick auf künftige Techniken wie die virtuelle Endoskopie gebracht. $N$ aturgemäß sind den für gastrointestinale $E$ rkrankungen relevanten diagnostischen Verfahren wie Endoskopie und Endosonographie, aber auch explorative Laparoskopie, viel Raum gewidmet.

Im folgenden A bschnitt werden alle A spekte der Pathologie über intraoperative Schnellschnittbefundung, pathologisches Staging, Beurteilung der Tumorregression nach präoperativer Therapie bis hin zur I mmunhistochemie und M olekularpathologie beschrieben. Als Überleitung zu den therapeutischen Prinzipien der Onkologie werden so wichtige und komplexe Themen wie Prognosefaktoren, Knochenmarkinfiltration, hereditäre Tumordispositionserkrankungen und präoperative Risikoabschätzung erläutert. Nachfolgend werden alle Prinzipien und Techniken der onkologischen Therapie wie onkologische Chirurgie, andere regionale Therapiekonzepte, Chemound Strahlentherapie präsentiert und derzeit noch experimentelle A nsätze wie die $G$ entherapie kurz abgehandelt.

U nter dem A bschnitt Q ualitätsmanagement folgt eine Z usammenfassung wichtiger Themen wie onkologische Konferenz, «second opinion», D okumentation, Komplikationserfassung und Psychoonkologie bzw. Lebensqualität.

I $\mathrm{m}$ zweiten Teil des Buches wird die Therapie gastrointestinaler Tumoren in anatomischer Reihenfolge abgehandelt. Die $\mathrm{K}$ apitel sind nach demselben Prinzip in E pidemiologie, Pathogenese, Pathologie, Prognose, Diagnostik und Staging, Therapie und $N$ achsorge eingeteilt.A lle A bschnitte werden ergänzt durch eigene E rfahrungen und wissenschaftliche Veröffentlichungen der Klinik, welche vor allem bei der Behandlung der Malignome des oberen $\mathrm{G}$ astrointestinaltrakts beeindruckend sind. Die Literaturzitate sind, wie bereits im Vorwort erwähnt, sehr subjektiv und nicht immer repräsentativ.

E rgänzt wird das Buch durch die Kapitel «Neuroendokrine Tumoren», «R etroperitoneale Weichteilsarkome» und «D er onkologische Chirurg in Kooperation mit U rologie und G ynäkologie».

A uf 432 Seiten wurden von insgesamt 67 Autoren die Prinzipien in der Therapie gastrointestinaler Tumoren der Chirurgischen Klinik und Poliklinik der Technischen U niversität $M$ ünchen aufgelistet. D er allgemeine $A$ bschnitt über die Prin-

\begin{tabular}{ll}
\hline KARGER & $@ 2000$ S. Karger G mbH , Freiburg \\
Fax +49 761 4520714 & A ccessible online at: \\
$\begin{array}{l}\text { E-mail Information@K arger.de } \\
\text { www.karger.com }\end{array}$ & www.karger.com/journals/onk
\end{tabular}


zipien der Diagnostik und Behandlungskonzepte scheint, wenngleich sehr übersichtlich und informativ, viel zu ausführlich und würde eher in ein Lehrbuch für Onkologie gehören. D ie A bhandlung der einzelnen Tumoren des $\mathrm{G}$ astrointestinaltrakts ist trotz der Vielzahl der A utoren sehr einheitlich aufgebaut. N aturgemäß wird den Tumoren von Ö sophagus, Magen und Pankreas mehr Platz einberaumt als z.B. dem Kolonkarzinom

Insgesamt ist das vorliegende Buch für onkologisch tätige Mediziner aller Fachbereiche mit dem Schwerpunkt gastrointestinale $M$ alignome von Interesse. A ufgrund der subjektiv ausgewählten Literaturzitate und der im Klinikum rechts der Isar gültigen Therapierichtlinien ist es nicht als Lehrbuch, sondern als Präsentation der eigenen E rfahrungen anzusehen.

G.V. Kornek, Wien

\section{Manual Maligne Lymphome, 6. Auflage}

H erausgeber: $B$. E mmerich

M ünchen, Z uckschwerdt, 2000, 192 S., D M 49,10

ISB N 3-88603-702-9

$M$ it der 6. A uflage dieses $M$ anuals liegt die überarbeitete Fassung eines von insgesamt 13 vom Tumorzentrum $M$ ünchen herausgegebenen Manualen mit Empfehlungen zur Diagnostik, Therapie und $\mathrm{N}$ achsorge maligner Erkrankungen vor.

Das Buch beginnt mit einer Präsentation epidemiologischer $D$ aten. E s schließt sich eine D arstellung der pathologisch-anatomischen $\mathrm{G}$ rundlagen an. G egenüberstellungen der gängigen $\mathrm{K}$ lassifikationen erleichtern hier die Orientierung. Das folgende Kapitel über immunzytologische Untersuchungen mag insbesondere für die Tätigkeit im durchflußzytometrischen $L$ abor hilfreich sein. $E$ in Kapitel über molekulargenetische A nalysen in der Lymphomdiagnostik, welches kursorisch die gängigen $M$ ethoden schildert, rundet den $G$ rundlagenteil $a b$. Daran anschließend wird auf 72 Seiten die Behandlung der großen Entitäten Morbus Hodgkin, Iymphozytische Lymphome und Immunozytom, follikuläre Lymphome, M antelzellLymphome und $\mathrm{M}$ arginalzonen-L ymphome, der hochmalignen
Non-Hodgkin-Lymphome sowie der peripheren T-Zell-Lymphome diskutiert. Das Vorwort des B andherausgebers Prof. Dr. E mmerich, daß «die E inbringung von Lymphompatienten in kontrollierte Therapiestudien generell zum empfehlen ist», findet hier seine konsequente U msetzung: In den jeweiligen $K$ apiteln werden nicht nur die aktuellen Konzepte der entsprechenden Studiengruppen dargestellt, sondern es werden dem L eser auch mit Verweis auf die E rgebnisse bisheriger Studien die $\mathrm{H}$ intergründe des jeweiligen Studiendesigns erklärt. A uch wenn diese K apitel keine einheitliche $\mathrm{G}$ liederung aufweisen, wirken sie in sich sehr homogen, bieten eine Fülle relevanter Informationen und sind auf dem neuesten Stand. Kontaktadressen der jeweiligen Studienzentralen, Therapieschemata und ein Verzeichnis der relevanten Literatur runden diese Kapitel ab.

E ntsprechend der wachsenden B edeutung der $\mathrm{H}$ ochdosistherapie mit autologer und allogener Blutstammzelltransplantation findet sich im A nschluß daran ein eigenes Kapitel zu dieser M odalität, in welchem auch zu pädiatrischen Transplantationen Stellung genommen wird. Die folgenden 40 Seiten des Buchs behandeln die Lymphomtherapie bei speziellen Kollektiven (im K indesalter, bei HIV-E rkrankung und Immunsuppression) oder bei besonderer Lokalisation (gastrointestinal, kutan, zentralnervös), und wenden sich somit vorrangig an den onkologischen Spezialisten. Nach Darstellung von Richtlinien zur Strahlentherapie und zu nuklearmedizinischen Verfahren endet der Text mit einem $\mathrm{K}$ apitel über $\mathrm{N}$ achsorgeuntersuchungen. E in umfangreiches Glossar und Stichwortverzeichnis stehen am Ende des Buchs.

Insgesamt liegt mit dieser N euauflage ein Werk vor, in dem die große A ufgabe gelungen ist, das $R$ elevante aus einigen tausend Seiten Studienprotokollen in ein griffiges Format zu bringen. $M$ an kann dieses $M$ anual jedem empfehlen, der Patienten mit malignen Lymphomen behandelt, sei es als jüngerer $A$ ssistent an einem hämatologischen Zentrum oder als Entscheidungsträger in einer nichtonkologischen $\mathrm{K}$ linik. A uch interessierten Internisten und A llgemeinärzten kann die L ektüre des $\mathrm{H}$ andbuchs sehr empfohlen werden, da es das für den nicht hämatoonkologisch Versierten scheinbar undurchschaubare Dickicht der Lymphomtherapie transparent macht.

W. Jung, M. P freundschuh, H omburg/Saar 